九州大学学術情報リポジトリ

Kyushu University Institutional Repository

\title{
Synthesis and Lateral Root-Inducing Activity of Novel 2-Piperidones with a 1,4-Benzodioxan Ring
}

Tsukada, Hidetaka

Laboratory of Pesticide Chemistry, Division of Bioresource and Bioenvironmental Sciences, Graduate School, Kyushu University

Yamada, Naotaka

Laboratory of Pesticide Chemistry, Division of Bioresource and Bioenvironmental Sciences, Graduate School, Kyushu University

Taniguchi, Eij i

Laboratory of Pesticide Chemistry, Division of Bioresource and Bioenvironmental Sciences, Graduate School, Kyushu University

Kuwano, Ei ichi

Laboratory of Pesticide Chemistry, Division of Bioresource and Bioenvironmental Sciences, Graduate School, Kyushu University

https://doi.org/10.5109/24334

出版情報：九州大学大学院農学研究院紀要. 44 (3/4)，pp.317-328，2000-02. Kyushu University バージョン：

権利関係 : 
J. Fac: Agr., Kyushu Univ., 44 (3-4), 317-328 (2000)

\title{
Synthesis and Lateral Root-Inducing Activity of Novel 2-Piperidones with a 1,4-Benzodioxan Ring
}

\author{
Hidetaka Tsukada, Naotaka Yamada, Eiji Taniguchi \\ and Eiichi Kuwano \\ Laboratory of Pesticide Chemistry: Division of Bioresource and Bioenvironmental \\ Sciences, Graduate School, Kyushu University, \\ Fukıoka 812-8581, Japan
}

(Received October 8, 1999 and accepted Novernber 5, 1999)

\begin{abstract}
A scries of novel $\mathrm{N}$-benzyl-2-piperidones having a 1,4-benzodioxan ring was synthesized and investigated for their activity to induce lateral roots in the lettuce seedlings. $N$-Benzyl-3-[1-(3,6-benzodioxanyl)-1-hydroxymethyl]-2-piperidone (23) induced $100 \%$ emergence of lateral roots at $100 \mathrm{ppm}$. There was no significant difference in activity between the erythro- and threo-23. The introduction of a benzyloxymethyl substituent at the 2-position on the 1,4-benzodioxan ring slightly increased the activity compared wiltu that of $\mathbf{2 3}$, showing some activity even at $1 \mathrm{ppm}$, while the activity was found to fall off sharply with a methoxy group adjacent to the 1,4-dioxarle ring.
\end{abstract}

\section{INTRODUCTION}

We have previously reported that a variety of $N$ benzyl-3-substituted-2-piperidones caused latcral root formation on lettuce seedlings (Tsukada ot al., 1999). The results from structure-activity relationship studies indicated that the substituent at the 3-position of 2-piperidone ring significantly affected the aclivity. Of these compounds tested, $N$-benzyl-3-[1-hydroxy-1-(4-quinolyl)methyl]-2-piperidone (1, Fig. 1) was the most effective for inducing lateral roots.

It is known that several natural products with a 1,4-benzodioxan ring such as haedoxan A (Taniguchi et al., 1989), cleomiscosin A (Ray et al., 1980), americanin (Woo et al., 1978) and silybin (Hansel et al., 1972) show some biological activity. It appears<smiles>O=C1C(C(O)c2ccnc3ccccc23)CCCN1Cc1ccccc1</smiles>

Fig. 1. Structure of $N$-benzyl-3[1-hydroxy-1-(4-quinolyl) methyl]-2-piperidone (1) 
that in these compounds the 1,4-benzodioxan moicty is essential for the activity. However, 1,4-benzodioxan derivatives have received little attention as molecules of potential agrochemical interest, and there has been no report in the literature of compounds with a 1,4-benzodioxan ring showing plant growth regulatory activity. We therefore synthesized a series of novel $N$-benzyl-2-piperidones possessing a 1,4-benzodioxan ring at the 3 -position and investigated their effects on the growth of lettuce seedlings. This paper describes their synthesis, lateral root-inducing activity and structure-activity relationships.

\section{MATERIALS $A$ ND METHODS}

\section{Synthesis}

All melting points (mp) are uncorrected. 'IH-NMR spectra were recorded on JNM-GX400 spectrometer with tetramethylsilane as an internal standard and all samples were prepared in deuterochloroform. Gravity column chromatography was carried out with Merck kiesegel 60 F254 (0.063-0.200 mm, 70-230 mesh ASTM) and Wakogel C-300 $(45-75 \mu \mathrm{m})$. TLC was performed on precoated $60 \mathrm{~F} 254$ silica gel plates $(0.25 \mathrm{~mm}$, $0.5 \mathrm{~mm}$, or $1 \mathrm{~mm}$ thickness, $20 \times 20 \mathrm{~cm})$ supplied by E. Merck.

4-Benzyloxy-3-hydroxybenzaldehyde (2).

To a solution of 3,4 dihydroxybenzaldchyde $(75 \mathrm{~g}, 0.54 \mathrm{~mol})$ in DMSO $(150 \mathrm{ml})$ was added sodium hydride $(60 \%$ in oil, washed with hexane, $22 \mathrm{~g}, 0.54 \mathrm{~mol})$ at $0^{\circ} \mathrm{C}$. After stirring for $1 \mathrm{hr}$ at room temperature, to the mixture was added benzyl chloride $(62 \mathrm{ml}$, $0.54 \mathrm{~mol}$ ). After stirring for $18 \mathrm{hr}$ at room temperature, the mixture was acidified with conc. $\mathrm{HCl}$ solution. The precipitate was collected by filtration and recrystallized from toluene to give pure 2 . Yield $30 \%$. White amorphous solid, mp $78-80^{\circ} \mathrm{C}$. ${ }^{1} \mathrm{H}-\mathrm{NMR} \delta: 1.65$ $(1 \mathrm{H}, \mathrm{br},-\mathrm{OH}), 5.21\left(2 \mathrm{H}, \mathrm{s},-\mathrm{O}-\mathrm{CH}{ }_{2}-\mathrm{Ph}\right), 7.04(1 \mathrm{H}, \mathrm{d}, \mathrm{J}=8 \mathrm{~Hz}, \mathrm{Ar}-\mathrm{H}), 7.38-7.47(7 \mathrm{H}, \mathrm{m}$, $\mathrm{Ar}-\mathrm{H}), 9.84(1 \mathrm{H}, \mathrm{s},-\mathrm{CHO})$.

3-Benzoylmethoxy-4-benzyloxybenzaldehyde (3)

To a solution of $\mathbf{2}(10 \mathrm{~g}, 44 \mathrm{mmol})$, potassium bicarbonate $(12.2 \mathrm{~g}, 88 \mathrm{mmol})$, and a catalytic amount of 18-crown-6 in $\mathrm{CH}_{3} \mathrm{CN}(200 \mathrm{ml})$ was added phenacyl bromide $(10.5 \mathrm{~g}$, $52.8 \mathrm{mmol}$ ). After stirring for $24 \mathrm{hr}$ at room temperature, the mixture was filtered through a ceiite pad. The filtrate was concentrated and to the residue was added ether $(500 \mathrm{ml})$ and water $(100 \mathrm{ml})$. The organic layer was separated, washed with $10 \%$ of $\mathrm{NaOH}$ solution, water, brine, and dried $\left(\mathrm{Na}_{4} \mathrm{SO}_{4}\right)$. Concentration of the solvent followed by recrystallization from ether gave pure 3. Yield $93 \%$. White amorphous solid, mp $107^{\circ} \mathrm{C}$. 'H-NMR $\delta: 5.31\left(2 \mathrm{H}, \mathrm{d}, \mathrm{J}=4 \mathrm{~Hz},-\mathrm{O}-\mathrm{CH}_{2}-\right), 5.46\left(2 \mathrm{H}, \mathrm{s},-\mathrm{O}-\mathrm{CH}_{2}-\right), 7.07-7.10(1 \mathrm{H}, \mathrm{m}$, $\mathrm{Ar}-\mathrm{H}), 7.30-7.31(1 \mathrm{H}, \mathrm{m}, \mathrm{Ar}-\mathrm{H}), 8.02-8.04(1 \mathrm{H}, \mathrm{m}, \mathrm{Ar}-\mathrm{H}), 9.85(1 \mathrm{H}, \mathrm{s},-\mathrm{CHO})$.

4-Benzyloxy-3-(2-hydroxy-2-phenylethoxy)benzaldehyde (4)

A solution of $\mathbf{3}(5 \mathrm{~g}, 14.4 \mathrm{mmol})$, ammoniun chloride $(1.6 \mathrm{~g}, 30 \mathrm{mmol})$, trimethyl orthoformate in THF : $\mathrm{MeOH}=3: 1(150 \mathrm{ml})$ was heated under refluxing for $8 \mathrm{hr}$. The reaction mixture was quenched by saturated aqueous $\mathrm{NaHCO}_{3}$ solution and concentrated. The residue was diluted with EtOAc $(500 \mathrm{ml})$. The organic layer was separated, washed with saturated aqueous $\mathrm{NaHCO}_{3}$ solution, brine, and dried $\left(\mathrm{Na}_{3} \mathrm{SO}_{4}\right)$. After removal of the solvent, the residue (crude acetal) was dissolved in $\mathrm{THF}: \mathrm{MeOH}=4: 1(100 \mathrm{ml})$ and to this solution was added sodium borohydride $\left(272 \mathrm{mg}, 7.2 \mathrm{mmol}\right.$ ) at $0^{\circ} \mathrm{C}$. After stirring for $1 \mathrm{hr}$ 
at $0^{\circ} \mathrm{C}$, the mixture was acidified with $2 \mathrm{~N} \mathrm{HCl}$ solution. After stirring for $2 \mathrm{hr}$, the reaction mixture was concentrated and the residue was diluted with Et.OAc $(300 \mathrm{ml})$. The organic layer was separated, washed with $10 \%$ of $\mathrm{NaOH}$ solution, water, brine, and dried $\left(\mathrm{Na}_{2} \mathrm{SO}_{1}\right)$. Concentration followed by column chromatography (silica gel, 50\% EtoAc in hexane) gave pure 4. Yield 90\%. Colorless oil. ' $\mathrm{H}-\mathrm{NMR} \delta: 3.39(1 \mathrm{H}, \mathrm{br},-\mathrm{OH}), 4.04-4.13$ $\left(1 \mathrm{H}, \mathrm{m},-\mathrm{O}-\mathrm{CH}_{2}-\right), 4.19-4.23\left(1 \mathrm{H}, \mathrm{m},-\mathrm{O}-\mathrm{CH}_{2}-\right), 5.10(1 \mathrm{H}, \mathrm{dd}, \mathrm{J}=3,9 \mathrm{~Hz},-\mathrm{CH}(\mathrm{OH})-), 5.18$ $\left(2 \mathrm{H}, \mathrm{s},-\mathrm{O}-\mathrm{CH}_{\mathrm{z}^{-}}\right), 7.02(1 \mathrm{H}, \mathrm{d}, \mathrm{J}=8 \mathrm{~Hz}, \mathrm{Ar}-\mathrm{H}), 7.25-7.53(7 \mathrm{H}, \mathrm{m}, \mathrm{Ar}-\mathrm{H}), 9.79(1 \mathrm{H}, \mathrm{s}$, $-\mathrm{CHO})$.

4-Hydroxy-3-(2-hydroxy-2-phenylethoxy)benzaldehyde (5)

A solution of $4(6.1 \mathrm{~g}, 17.5 \mathrm{mmol})$ and palladium on carbon ( $5 \%$ on carbon, $0.6 \mathrm{~g})$ in EtOAc: : $\mathrm{MeOH}=1: 1(50 \mathrm{ml})$ was stirred under hydrogen gas. After stirring for $24 \mathrm{hr}$, the mixture was filtered through a celite pad and the filtrate was concentrated. Recrystallization from diisopropyl ether gave pure alcohol $5(1.85 \mathrm{~g}, 41 \%)$. White amorphous solid, mp $114^{\circ} \mathrm{C}$. ' $\mathrm{H}-\mathrm{NMR} \delta: 1.84(1 \mathrm{H}, \mathrm{br},-\mathrm{OH}), 4.07-4.12\left(3 \mathrm{H}, \mathrm{m},-\mathrm{O}-\mathrm{CH}_{2}{ }^{-}\right.$, $-\mathrm{OH}), 4.19-4.22\left(1 \mathrm{H}, \mathrm{m},-\mathrm{O}^{-} \mathrm{CH}_{2}{ }^{-}\right), 5.13(1 \mathrm{H}, \mathrm{dd}, \mathrm{J}=3,9 \mathrm{~Hz},-\mathrm{C} \underline{\mathrm{H}}(\mathrm{OH})-), 7.01(1 \mathrm{H}, \mathrm{d}, \mathrm{J}=8$ $\mathrm{Hz}, \mathrm{Ar}-\mathrm{H}), 7.34-7.42(6 \mathrm{H}, \mathrm{m}, \mathrm{Ar}-\mathrm{H}), 9.73(1 \mathrm{H}, \mathrm{s},-\mathrm{CHO})$.

2-Phenyl-1,4-benzodioxan-6-carbaldehyde (6)

A solution of 5 ( $100 \mathrm{mg}, 0.38 \mathrm{mmol})$ and a catalytic amount of $p$-toluenesulfonic acid in toluene $(30 \mathrm{ml})$ was heated under refluxing for $16 \mathrm{hr}$. After cooling, to the mixture was added $\mathrm{Et}_{3} \mathrm{~N}(50 \mathrm{ml})$. Concentration followed by column chromatography (silica gel, $25 \%$ EtOAc in hexane) gave pure 6. Yield $58 \%$. White amorphous solid, mp $75-77^{\circ} \mathrm{C}$. 'H-NMR $\delta: 4.06\left(1 \mathrm{H}, \mathrm{dd}, \mathrm{J}=9 \mathrm{~Hz}, 12 \mathrm{~Hz},-\mathrm{O}^{-} \mathrm{CH}_{\mathbf{z}^{-}}\right), 4.43\left(1 \mathrm{H}, \mathrm{dd}, \mathrm{J}=9,12 \mathrm{~Hz},-\mathrm{O}^{-} \mathrm{CH}_{2}^{-}\right)$, $5.20(1 \mathrm{H}, \mathrm{dd}, \mathrm{J}=2.9 \mathrm{~Hz},-\mathrm{O}-\mathrm{CH}-), 7.10(1 \mathrm{H}, \mathrm{d}, \mathrm{J}=8.3 \mathrm{~Hz}, \mathrm{Ar}-\mathrm{H}), 7.34-7.52(7 \mathrm{H}, \mathrm{m}, \mathrm{Ar}-\mathrm{H})$, $9.86(1 \mathrm{H}, \mathrm{s},-\mathrm{CHO})$.

4-Benzyloxy-3-(2,3-epoxypropyloxy)benzaldehyde (7)

To a suspension of sodium hydride ( $60 \%$ in oil, washed with hexane, $17.2 \mathrm{~g}, 0.43 \mathrm{~mol}$ ) in DMF $(250 \mathrm{ml})$ was added $2(75 \mathrm{~g}, 0.33 \mathrm{mmol})$ at $0{ }^{\circ} \mathrm{C}$. After stirring for $1 \mathrm{hr}$ at room temperature, to the mixture was added 1-bromo-2,3-epoxypropane $(34 \mathrm{ml}, 0.40 \mathrm{~mol})$. After stirring for $18 \mathrm{hr}$ at room temperature, the mixture was quenched with water $(200 \mathrm{ml})$, and diluted with ether $(1000 \mathrm{ml})$ The organic layer was separated, washed with water, $5 \% \mathrm{NaOH}$ solution, brine and dried $\left(\mathrm{Na}_{2} \mathrm{SO}_{4}\right)$. After concentration of the solvent, the residue was recrystallized from $\mathrm{EtOH}$ to give pure 7 . Yield $44 \%$. Colorless crystal, mp $94-96{ }^{\circ} \mathrm{C} .{ }^{1} \mathrm{H}-\mathrm{NMR} \delta: 2.79-2.80\left(1 \mathrm{H}, \mathrm{q}, \mathrm{J}=2 \mathrm{~Hz},-\mathrm{CH}(\mathrm{O}) \mathrm{CH}_{2}\right), 2.91(1 \mathrm{H}, \mathrm{t}, \mathrm{J}=4 \mathrm{~Hz}$, $\left.-\mathrm{CH}(\mathrm{O}) \mathrm{CH}_{2}\right), 3.40-3.44\left(1 \mathrm{H}, \mathrm{m},-\mathrm{CH}(\mathrm{O}) \mathrm{CH}_{2}\right), 4.05-4.09\left(1 \mathrm{H}, \mathrm{dd}, \mathrm{J}=5,9 \mathrm{~Hz},-\mathrm{O}-\mathrm{CH}_{2}-\right)$, 4.34-4.40 (1H, dd, $\left.J=3,11 \mathrm{~Hz},-0-\mathrm{CH}_{2}-\right), 5.27\left(1 \mathrm{H}, \mathrm{s},-0-\mathrm{CH}_{2}-\right), 7.00(1 \mathrm{H}, \mathrm{d}, \mathrm{J}=8.3 \mathrm{~Hz}$, $\mathrm{Ar}=\mathrm{H}), 7.32-7.46(\mathrm{~m}, 7 \mathrm{H}, \mathrm{Ar}-\mathrm{H}), 9.83(1 \mathrm{H}, \mathrm{s},-\mathrm{CHO})$.

3-(2,3-Epoxypropyloxy)-4-hydroxybenzaldehyde (8)

A mixture of $7(42.5 \mathrm{~g}, 0.14 \mathrm{~mol})$ and palladium on carbon $(5 \%$ on carbon, $4.2 \mathrm{~g}$ ) in EtOAc $(250 \mathrm{ml})$ was stirred under hydrogen gas. After stirring for $2 \mathrm{hr}$, the mixture was filtered through a celite pad and the filtrate was concentrated. Separation by column chromatography (silica gel, 50\% EtOAc in hexane) gave pure 8. Yield $90 \%$. White amorphous solid, mp $71-72^{\circ} \mathrm{C}$. 'H-NMR $\delta: 2.83-2.84\left(1 \mathrm{H}, \mathrm{m},-\mathrm{CH}(\mathrm{O}) \mathrm{CH}_{2}\right), 2.97-2.99$ $\left(1 \mathrm{H}, \mathrm{m},-\mathrm{CH}(\mathrm{O}) \mathrm{C}_{2}\right), 3.40-3.44\left(1 \mathrm{H}, \mathrm{m},-\mathrm{CH}(\mathrm{O}) \mathrm{CH}_{2}\right), 4.04(1 \mathrm{H}, \mathrm{dd}, \mathrm{J}=6,11 \mathrm{~Hz}$, $\left.-\mathrm{O}^{-} \mathrm{CH}_{2}-\right), 4.44\left(1 \mathrm{H}, \mathrm{dd}, \mathrm{J}=2,11 \mathrm{~Hz},-\mathrm{O}-\mathrm{CH}_{2}^{-}\right), 6.64(1 \mathrm{H}, \mathrm{s},-\mathrm{OH}), 7.07(1 \mathrm{H}, \mathrm{d}, \mathrm{J}=8.3 \mathrm{~Hz}$, $\mathrm{Ar}-\mathrm{H}), 7.43-7.48(2 \mathrm{H}, \mathrm{m}, \mathrm{Ar}-\mathrm{H}), 9.82(1 \mathrm{H}, \mathrm{s},-\mathrm{CHO})$. 


\section{2-Hydroxymethyl-1,4-benzodioxan-6-carbaldehyde (9)}

A mixture of $\mathbf{8}(38 \mathrm{~g}, 0.14 \mathrm{~mol})$, potassium bicarbonate $(55.3 \mathrm{~g}, 0.4 \mathrm{~mol})$, and 18-crown-6 (3.8g) in $\mathrm{CH}_{3} \mathrm{CN}(250 \mathrm{ml})$ was stirred for $16 \mathrm{hr}$ at room temperature. The mixture was filtered through a celite pad. Concentration of the filtrate followed by column chromatography (silica gel, $50 \%$ EtOAc in hexane) gave pure 9. Yield $43 \%$. White amorphous solid, mp 59-60 ${ }^{\circ} \mathrm{C}$. ' $\mathrm{H}-\mathrm{NMR} \delta: 1.95(1 \mathrm{H}, \mathrm{t}, \mathrm{J}=6 \mathrm{~Hz},-\mathrm{OH}), 3.86-4.06$ $\left(2 \mathrm{H}, \mathrm{m},-\mathrm{O}-\mathrm{CH}_{2}-\right), 4.14-4.19\left(1 \mathrm{H}, \mathrm{m},-\mathrm{O}-\mathrm{CH}_{2}-\mathrm{C} \underline{\mathrm{H}}-\mathrm{O}-\right), 4.34-4.39\left(2 \mathrm{H}, \mathrm{m},-\mathrm{CH}_{2}-\mathrm{OH}\right), 7.02$ $(1 \mathrm{H}, \mathrm{d}, \mathrm{J}=9 \mathrm{~Hz}, \mathrm{Ar}-\mathrm{H}), 7.42-7.44(2 \mathrm{H}, \mathrm{m}, \mathrm{Ar}-\mathrm{H}), 9.83(1 \mathrm{H}, \mathrm{s},-\mathrm{CHO})$.

2-Methoxymethyl-1,4-benzodioxan-6-carbaldehyde (10)

A mixture of 9 ( $2 \mathrm{~g}, 10.3 \mathrm{mmol})$, sodium hydroxide $(4.1 \mathrm{~g}, 103 \mathrm{mmol})$, methyl iodide $(103 \mathrm{mmol})$ and tetrabutylammonium hydrogen sulfate $(1.77 \mathrm{~g}, 5.2 \mathrm{mmol})$ in water-dichloromethane $=1: 1(100 \mathrm{ml})$ was stirred for $72 \mathrm{hr}$ at room temperature. The aqueous layer was separated and the product was extracted with EtOAc $(400 \mathrm{ml})$. The combined organic layer was washed with water, brine and dried $\left(\mathrm{Na}_{2} \mathrm{SO}_{4}\right)$. Concentration followed by column chromatography (silica gel, 25\% EtOAc in hexane) gave pure 10. Yield $84 \%$. Colorless oil. 'H-NMR $\delta: 3.44\left(3 \mathrm{H}, \mathrm{s},-\mathrm{OCH}_{3}\right), 3.61-3.71\left(2 \mathrm{H}, \mathrm{m},-\mathrm{O}-\mathrm{CH}_{2}{ }^{-}\right)$, $4.08-4.13\left(1 \mathrm{H}, \mathrm{m},-\mathrm{O}-\mathrm{CH}_{3}-\mathrm{CH}^{-} \mathrm{O}^{-}\right), 4.30-4.42\left(2 \mathrm{H}, \mathrm{m},-\mathrm{O}-\mathrm{CH}_{2}-\right), 6.90-7.03(1 \mathrm{H}, \mathrm{m}$, $\mathrm{Ar}-\mathrm{H}), 7.40-7.42(2 \mathrm{H}, \mathrm{m}, \mathrm{Ar}-\mathrm{H}), 9.82(1 \mathrm{H}, \mathrm{s},-\mathrm{CHO})$.

Compounds 11 and 12 were prepared in the same manner as 10 by using the corresponding alkyl bromide instead of the methyl iodide.

2-Ethoxymethyl-1,4-benzodioxan-6-carbaldehyde (11). Yield 82\%. Colorless oil. 'H-NMR $\delta$ : $1.14-1.20\left(3 \mathrm{H}, \mathrm{m},-\mathrm{CH}_{3}\right), 3.48-3.68\left(3 \mathrm{H}, \mathrm{m},-\mathrm{O}-\mathrm{CH}_{2}-,-\mathrm{CH}-\mathrm{O}-\right), 4.27-4.35$ $\left.\left(2 \mathrm{H}, \mathrm{m},-\mathrm{O}-\mathrm{CH}_{2}-\right), 4.08-4.13\left(1 \mathrm{H}, \mathrm{m},-\mathrm{O}-\mathrm{CH}_{3}-\mathrm{CH}^{-}-\right)^{-}\right), 6.94(1 \mathrm{H}, \mathrm{d}, \mathrm{J}=9 \mathrm{~Hz}, \mathrm{Ar}-\mathrm{H})$, $7.33-7.35(2 \mathrm{H}, \mathrm{m}, \mathrm{Ar}-\mathrm{H}), 9.75(1 \mathrm{H}, \mathrm{s},-\mathrm{CHO})$.

2-Benzyloxymethyl-1,4-benzodiaxan-6-carbaldehyde (12). Yield 95\%. Coloriess oil. ${ }^{1} \mathrm{H}-\mathrm{NMR} \delta: 3.66\left(1 \mathrm{H}, \mathrm{m}, \mathrm{J}=6,10 \mathrm{~Hz},-\mathrm{O}-\mathrm{CH}_{2}-\right), 3.73(1 \mathrm{H}$, dd, J = 5, $10 \mathrm{~Hz}$, $\left.-\mathrm{O}-\mathrm{CH}_{2}-\right), 4.11-4.27\left(1 \mathrm{H}, \mathrm{m},-\mathrm{O}-\mathrm{CH}_{2}-\mathrm{CH}-\mathrm{O}-\right), 4.31-4.42\left(2 \mathrm{H}, \mathrm{m},-\mathrm{O}-\mathrm{CH}_{2}-\right), 6.98(1 \mathrm{H}, \mathrm{d}$, $\mathrm{J}=9 \mathrm{~Hz}, \mathrm{Ar}-\mathrm{H}), 7.25-7.40(7 \mathrm{H}, \mathrm{m}, \mathrm{Ar}-\mathrm{H}), 9.79(1 \mathrm{H}, \mathrm{s},-\mathrm{CHO})$.

6-Methoxy-1,4-benzodioxan (14)

To a mixture of $m$-chloroperbenzoic acid ( $80 \%$ in water, $25 \mathrm{~g}, 145 \mathrm{mmol}$ ), and potassium fluoride $(10.5 \mathrm{~g}, 181 \mathrm{mmol})$ in $\mathrm{CH}_{2} \mathrm{Cl}_{2}$ was added 1,4-benzodioxan-6-carbaldehyde $(10 \mathrm{~g}, 72.4 \mathrm{mmol})$ in $\mathrm{CH}_{2} \mathrm{Cl}:(100 \mathrm{ml})$ at $0^{\circ} \mathrm{C}$. After stirring for $40 \mathrm{hr}$ at $0^{\circ} \mathrm{C}$ to room temperature, the mixture was filtered through a celite pad and the filtrate was concentrated. To the residue was added $50 \% \mathrm{NaOH}$ solution $(100 \mathrm{ml})$ and the mixture was stirred for $2 \mathrm{hr}$. To the mixture was added ether $(500 \mathrm{ml})$ and the aqueous layer was separated, acidified with conc. $\mathrm{HCl}$ solution. The product was extracted with ether $(500 \mathrm{ml})$. The organic layer was separated, washed with saturated aqueous $\mathrm{NaHCO}_{i}$ solution, brine, and dried $\left(\mathrm{Na}_{3} \mathrm{SO}_{4}\right)$. Concentration of the solvent afforded crude phenol (6-hydroxy-1,4-benzodioxan). A mixture of crude phenol, methyl iodide ( $31 \mathrm{~g}$, $217 \mathrm{mmol})$ and potassium carbonate $(30 \mathrm{~g}, 217 \mathrm{mmol})$ in acetone $(250 \mathrm{ml})$ was heated under refluxing for $6 \mathrm{hr}$. The mixture was filtered through a celite pad and concentrated. The resulting residue was purified by column chromatography (silica gel, 25\% EtOAc in hexane) to give pure 14. Yield $64 \%$. Colorless oil. 'H-NMR $\delta: 3.73\left(3 \mathrm{H}, \mathrm{s},-\mathrm{O}-\mathrm{CH}_{3}\right), 4.19$ $\left(4 \mathrm{H}, \mathrm{m},-\mathrm{O}-\mathrm{C} \mathrm{H}_{2}-\mathrm{CH}_{2}-\mathrm{O}-\right), 6.40-6.45(2 \mathrm{H}, \mathrm{m}, \mathrm{Ar}-\mathrm{H}), 6.76-6.79(1 \mathrm{H}, \mathrm{m}, \mathrm{Ar}-\mathrm{H})$.

6-Methoxy-1,4-benzodioxan-7-carbaldehyde (15) 
To a solution of $\mathbf{1 4}(7.5 \mathrm{~g}, 45.1 \mathrm{mmol})$ in DMF $(50 \mathrm{ml})$ was added $\mathrm{POCl}_{3}(13 \mathrm{ml}$, $135 \mathrm{mmol}$ ) at $0^{\circ} \mathrm{C}$. After stirring for $5 \mathrm{hr}$ at $100^{\circ} \mathrm{C}$, the mixture was poured into ice-cooled water $(500 \mathrm{ml})$. The solution was neutralized with potassium bicarbonate and extracted with ether $(2000 \mathrm{ml})$. The organic layer was separated, washed with saturated aqueous $\mathrm{NaHCO}_{3}$ solution, brine, and dried $\left(\mathrm{Na}_{2} \mathrm{SO}_{4}\right)$. After concentration of the solvent, the residue was recrystallized from diisopropyl ether to give pure $\mathbf{1 5}$. Yield $78 \%$. White amorphous solid, $\mathrm{mp} 116^{\circ} \mathrm{C}$. ${ }^{1} \mathrm{H}-\mathrm{NMR} \delta: 3.84\left(3 \mathrm{H}, \mathrm{s},-\mathrm{O}-\mathrm{CH}_{3}\right), 4.21-4.24(2 \mathrm{H}, \mathrm{m}$, $\left.-\mathrm{O}-\mathrm{CH}_{2}-\mathrm{CH}_{2}-\mathrm{O}-\right), 4.31-4.33\left(2 \mathrm{H}, \mathrm{m},-\mathrm{O}-\mathrm{CH}_{2}-\mathrm{CH}_{2}-\mathrm{O}-\right), 6.46(1 \mathrm{H}, \mathrm{s}, \mathrm{Ar}-\mathrm{H}), 7.36(1 \mathrm{H}, \mathrm{m}$, $\mathrm{Ar}-\mathrm{H}), 10.27(1 \mathrm{H}, \mathrm{s},-\mathrm{CHO})$.

Methyl 5-methoxy-1,4-benzodioxan-7-carboxylate (17)

To a solution of methyl gallate ( $20 \mathrm{~g}$ : $0.11 \mathrm{~mol}$ ) in $10 \%$ aqueous sodium terraborate solution $(800 \mathrm{ml})$ was added aqueous $\mathrm{NaOH}$ solution ( $26 \mathrm{~g}$ in water) and dimethyl sulfate $(60 \mathrm{ml})$ during $3 \mathrm{hr}$. After stirring for $12 \mathrm{hr}$ at room temperature, the mixture was acidified with conc. $\mathrm{HCl}$ solution and extracted with ether $(2000 \mathrm{ml})$. The organic layer was separated, washed with water, brine, and dried $\left(\mathrm{Na}_{3} \mathrm{SO}_{4}\right)$. Concentration of the solvent gave crude 5-methoxycarbonyl-3-methoxycatechol (16). A mixture of crude 16, 1,2 -dibromoethane $(41 \mathrm{~g}, 0.22 \mathrm{~mol})$, potassium carbonate $(76 \mathrm{~g}, 0.55 \mathrm{~mol})$ in acetone $(250 \mathrm{ml})$ was heated under refluxing for $6 \mathrm{hr}$. The mixture was filtered through a celite pad and the filtrate was concentrated. The residue was dissolved in ether $(1000 \mathrm{ml})$, and water $(200 \mathrm{ml})$. The organic layer was separated, washed with $10 \% \mathrm{NaOH}$ solution, water, brine, and dried $\left(\mathrm{Na}_{2} \mathrm{SO}_{4}\right)$. After concentration of the solvent, the residue was recrystallized from diisopropyl ether to afford pure 17. Yield $21 \%$. Colorless amorphous solid, mp $107^{\circ} \mathrm{C}$. ' $\mathrm{H}-\mathrm{NMR} \delta$ : $3.89\left(3 \mathrm{H}, \mathrm{s},-\mathrm{O}-\mathrm{CH}_{3}\right), 3.93\left(3 \mathrm{H}, \mathrm{s},-\mathrm{O}^{-} \mathrm{CH}_{3}\right), 4.27-4.29(2 \mathrm{H}$, $\left.\mathrm{m},-\mathrm{O}-\mathrm{CH}_{2}-\mathrm{CH}_{2}-\mathrm{O}-\right), 4.37-4.38\left(2 \mathrm{H}, \mathrm{m},-\mathrm{O}-\mathrm{CH}_{2}-\mathrm{CH}_{2}-\mathrm{O}^{-}\right), 7.20(1 \mathrm{H}, \mathrm{d}, \mathrm{J}=2 \mathrm{~Hz}, \mathrm{Ar}-\mathrm{H})$, 7.23-7.30 (2H, m, Ar-H).

\section{5-Methoxy-1,4-benzodioxan-7-carbaldehyde (19)}

To a mixture of lithium aluminum hydride $(4.25 \mathrm{~g}, 112 \mathrm{mmol})$ was added $17(5.0 \mathrm{~g}$, $22.3 \mathrm{mmol})$ in $\mathrm{THF}(50 \mathrm{~mJ})$ at $0^{\circ} \mathrm{C}$. After stirring for $1.5 \mathrm{hr}$ at room temperature, the mixture was quenched with saturated aqueous $\mathrm{MgSO}_{4}$ solution, and then potassium carbonate $(50 \mathrm{~g})$ was added. After stirring for 30 minutes at room temperature, the mixture was filtered through a celite pad and the filtrate was concentrated. The residue was dissolved in ELOAC $(500 \mathrm{ml})$ and water $(100 \mathrm{ml})$. The organic layer was separated, washed with brine, and dried $\left(\mathrm{Na}_{2} \mathrm{SO}_{4}\right)$. Concentration of the solvent afforded crude alcohol 18. To a solution of oxalyl chloride in $\mathrm{CH}_{2} \mathrm{Cl}_{2}(50 \mathrm{ml})$ was added DMSO $(2.38 \mathrm{ml}$, $33.5 \mathrm{mmol})$ in $\mathrm{CH}_{2} \mathrm{Cl}_{2}(10 \mathrm{ml})$ at $-78^{\circ} \mathrm{C}$. After stirring for 30 minutes at $-78^{\circ} \mathrm{C}$, to the mixlure was added crude 18 in $\mathrm{CH}_{2} \mathrm{Cl},(50 \mathrm{ml})$ at $-78^{\circ} \mathrm{C}$. The solution was stirred for $2 \mathrm{hr}$ at $-78^{\circ} \mathrm{C}$ and then tricthylamine $(15.6 \mathrm{ml}, 112 \mathrm{mmol})$ was added at $-78^{\circ} \mathrm{C}$. The solution was allowed to warm to room temperature, and then diluted with EtOAc $(300 \mathrm{ml})$ and water $(100 \mathrm{ml})$. The organic layer was separated, washed with brine, and dried $\left(\mathrm{Na}_{2} \mathrm{SO}_{4}\right)$. Concentration followed by recrystallization from diisopropyl ether gave pure 19. Yield $66 \%$. Colorless needle, mp $83^{\circ} \mathrm{C}$. 'H-NMR $\delta: 3.95\left(3 \mathrm{H}, \mathrm{s},-\mathrm{O}-\mathrm{CH}_{3}\right), 4.30-4.32(2 \mathrm{H}, \mathrm{m}$, $\left.-\mathrm{O}-\mathrm{CH}_{2}-\mathrm{CH}_{2}-\mathrm{O}-\right), 4.40-4.42\left(2 \mathrm{H}, \mathrm{m},-\mathrm{O}-\mathrm{CH}_{2}-\mathrm{CH}_{2}-\mathrm{O}-\right), 7.08(1 \mathrm{H}, \mathrm{s}, \mathrm{Ar}-\mathrm{H}), 7.26(1 \mathrm{H}, \mathrm{s}$, $\mathrm{Ar}-\mathrm{H}), 9.79(1 \mathrm{H}, \mathrm{s},-\mathrm{CHO})$.

5-Methoxy-1,4-benzodioxan-6-carbaldehyde (21)

A mixture of 3-methoxycatechol ( $10 \mathrm{~g}, 71.4 \mathrm{mmol}$ ), 1,2-dibromoethane (20.0 g, 
$0.107 \mathrm{~mol})$, potassium carbonate $(49.3 \mathrm{~g}: 357 \mathrm{~mol})$ in acetone $(250 \mathrm{ml})$ was heated under refluxing for $6 \mathrm{hr}$. The mixture was filtered through a celite pad and the filtrate was concentrated. The residue was dissolved in ether $(1000 \mathrm{ml})$, and water $(200 \mathrm{ml})$. The organic layer was separated, washed with $10 \% \mathrm{NaOH}$ solution, watcr, brine: and dried $\left(\mathrm{Na}_{2} \mathrm{SO}_{4}\right)$. Concentration of the solvent gave crude 5-methoxy-1,4-benzodioxan (20). To a solution of crude $\mathbf{2 0}$ in DMF (30 ml) was added $\mathrm{POCl}_{4}(4.0 \mathrm{ml}, 84.8 \mathrm{mmol})$ at $0^{\circ} \mathrm{C}$. After stirring for $5 \mathrm{hr}$ at $100^{\circ} \mathrm{C}$, the mixture was poured into ice-cooled water $(500 \mathrm{ml})$. The mixture was neutralized with potassium bicarbonate and extracted with ether $(500 \mathrm{ml})$. The organic layer was separated, washed with saturated aqueous $\mathrm{NaHCO}_{3}$ solution, brine, dricd $\left(\mathrm{Na}_{2} \mathrm{SO}_{4}\right)$, and concentrated. The residue was recrystallized from disopropyl ether to afford pure 21 . Yield $46 \%$. White amorphous solid, mp $134-137^{\circ} \mathrm{C}$. 'H-NMR $\delta: 3.95\left(3 \mathrm{H}, \mathrm{s},-\mathrm{O}-\mathrm{CH}_{3}\right), 4.38-4.41\left(4 \mathrm{H}, \mathrm{m},-\mathrm{O}-\mathrm{CH}_{2}-\mathrm{CH}_{2}-\mathrm{O}-\right), 6.59(1 \mathrm{H}, \mathrm{d}, \mathrm{J}=9$ $\mathrm{Hz}, \mathrm{Ar}-\mathrm{H}), 7.43(1 \mathrm{H}, \mathrm{d}, \mathrm{J}=9, \mathrm{Ar}-\mathrm{H}), 10.21(1 \mathrm{H}, \mathrm{s},-\mathrm{CHO})$.

N-Benzyl-3-[1-hydroxy-1-(3,6-benzodioxanyl)methyl-2-piperidone (23)

To a solution of diisopropylamine $(1.1 \mathrm{ml}, 7.92 \mathrm{mmol})$ in THF $(100 \mathrm{ml})$ was added $n$-butyllithium ( $4.95 \mathrm{ml}, 1.6 \mathrm{M}$ solution in hexane) at $-78^{\circ} \mathrm{C}$ under nitrogen gas. After stirring for 15 minutes, a solution of $N$-benzyl-2-piperidone $(1 \mathrm{~g}, 5.28 \mathrm{mmol})$ in $\mathrm{THF}^{2}$ ( $2 \mathrm{mb}$ ) was added dropwise, and then the mixture was stirred at $-78^{\circ} \mathrm{C}$ for 30 minutes. To the mixture was added a solution of 1 ,4-benzodioxan-6-carbaldehyde (1.04 g, 6.34 mmol) in THF ( $2 \mathrm{ml}$ ) was added at $-78^{\circ} \mathrm{C}$. After stirring at $-78^{\circ} \mathrm{C}$ for $3 \mathrm{hr}$, to the mixture was added saturated aqueous $\mathrm{NH}_{4} \mathrm{Cl}$ solution $(50 \mathrm{ml})$ and EtOAc $(100 \mathrm{ml})$. The organic layer was separated, washed with brine, and dried $\left(\mathrm{Na}_{2} \mathrm{SO}_{4}\right)$. Concentration followed by column chromatography (silica gel, 50\% EtOAc in hexane) gave pure $\mathbf{2 3}$ which was a diastereomeric mixture of erythro and threo (1:1). Yield 88\%. ${ }^{1} \mathrm{H}-\mathrm{NMR} \delta: 1.23-1.28$ $(2 \mathrm{H}, \mathrm{m}, \beta-\mathrm{H}, \gamma-\mathrm{H}), 1.56-1.75(2 \mathrm{H}, \mathrm{m}, \beta-\mathrm{H}, \gamma-\mathrm{H}),, 2.49-2.56(0.5 \mathrm{H}, \mathrm{m}, \alpha-\mathrm{H}), 2.80-2.83$ $(0.5 \mathrm{H}, \mathrm{m}, \alpha-\mathrm{H}), 3.07-3.11(1 \mathrm{H}, \mathrm{m}, \delta-\mathrm{H}), 3.20-3.23(1 \mathrm{H}, \mathrm{m}, \delta-\mathrm{H}), 4.24\left(2 \mathrm{H}, \mathrm{s},-\mathrm{O}-\mathrm{CH}_{2}-\right)$, $4.44-4.78\left(3 \mathrm{H}, \mathrm{m},-\mathrm{C} \underline{\mathrm{H}}(\mathrm{O} \underline{\mathrm{H}})^{-},-\mathrm{CH}_{2}-\mathrm{Ph}\right), 5.14(0.5 \mathrm{H}, \mathrm{dd}, \mathrm{J}=4,6 \mathrm{~Hz},-\mathrm{CH}(\mathrm{OH})-), 6.45$ $(0.5 \mathrm{H}, \mathrm{s},-\mathrm{OH}), 6.79-6.91(2 \mathrm{H}, \mathrm{m}, \mathrm{Ar}-\mathrm{H}), 7.18-7.34(6 \mathrm{H}, \mathrm{m}, \mathrm{Ar}-\mathrm{H})$.

eryithro- 23 white amorphous solid, mp $132^{\circ} \mathrm{C}$. 'H-NMR $\delta: 1.56-1.74(4 \mathrm{H}, \mathrm{m}, \beta-\mathrm{H}$, $\gamma-\mathrm{H}), 2.79-2.82(1 \mathrm{H}, \mathrm{m}, \alpha-\mathrm{H}), 3.07-3.13(2 \mathrm{H}, \mathrm{m}, \delta-\mathrm{H}), 4.25\left(4 \mathrm{H}, \mathrm{s},-\mathrm{O}^{-} \mathrm{CH}_{2}-\mathrm{CH},-\mathrm{O}^{-}\right)$, $4.45\left(1 \mathrm{H}, \mathrm{d}, \mathrm{J}=15,-\mathrm{CH}_{2}-\mathrm{Ph}\right), 4.69-4.70(1 \mathrm{H}, \mathrm{m},-\mathrm{OH}), 4.76\left(1 \mathrm{H}, \mathrm{d}, \mathrm{J}=15 \mathrm{~Hz},-\mathrm{CH}_{2}-\mathrm{Ph}\right)$, $5.15(1 \mathrm{H}, \mathrm{dd}, \mathrm{J}=3,6 \mathrm{~Hz},-\mathrm{CH}(\mathrm{OH})-), 6.81(\mathrm{~s}, 2 \mathrm{H}, \mathrm{Ar}-\mathrm{H}), 6.90(1 \mathrm{H}, \mathrm{s}, \mathrm{Ar}-\mathrm{H}), 7.18-7.36$ $(5 \mathrm{H}, \mathrm{m}, \mathrm{Ar}-\mathrm{H})$. Anal. Found: $\mathrm{C}, 71.28 ; \mathrm{H}, 6.59 ; \mathrm{N}, 3.98 \%$. Calcd. for $\mathrm{C}_{12} \mathrm{H}_{15} \mathrm{NO}: \mathrm{C}, 71.37$; $\mathrm{H}, 6.56 ; \mathrm{N}, 3.96 \%$.

threo- 23 white amorphous solid, mp $132-133^{\circ} \mathrm{C}$. 'H-NMR $\delta: 1.23-1.38(2 \mathrm{H}, \mathrm{m}, \beta$ $-\mathrm{H}), \mathrm{l} .72-1.79(2 \mathrm{H}, \mathrm{m}, \gamma-\mathrm{H}), 2.49-2.55(1 \mathrm{H}, \mathrm{m}, \alpha-\mathrm{H}), 3.20-3.23(2 \mathrm{H}, \mathrm{m}, \delta-\mathrm{H}), 4.24(4 \mathrm{H}$, $\left.\mathrm{s},-\mathrm{O}-\underline{\mathrm{CH}}_{3}-\mathrm{CH}_{2}-\mathrm{O}-\right), 4.54\left(1 \mathrm{H}, \mathrm{d}, \mathrm{J}=15 \mathrm{~Hz},-\mathrm{CH}_{2}-\mathrm{Phl}\right), 4.64(1 \mathrm{H}, \mathrm{d}, \mathrm{J}=10 \mathrm{~Hz},-\mathrm{CH}(\mathrm{OH})-)$, $4.69\left(1 \mathrm{H}, \mathrm{d}, \mathrm{J}=15 \mathrm{~Hz},-\mathrm{CH}_{2}-\mathrm{Ph}\right), 6.45(1 \mathrm{H}, \mathrm{s},-\mathrm{OH}), 6.83-6.96(3 \mathrm{H}, \mathrm{m}, \mathrm{Ar}-\mathrm{II}), 7.18-7.37$ $(5 \mathrm{H}, \mathrm{m}, \mathrm{Ar}-\mathrm{H})$. Anal. Found: $\mathrm{C}, 71.37 ; \mathrm{H}, 6.63 ; \mathrm{N}, 4.04 \%$. Calcd. for $\mathrm{C}_{12} \mathrm{H}_{15} \mathrm{NO}: \mathrm{C}, 71.37$; $\mathrm{H}, 6.56 ; \mathrm{N}, 3.96 \%$.

Compounds 24-30 were prepared in the same manner as $\mathbf{2 3}$ by using the corresponding substituted-1,4-benzodioxan-6-carbaldehydes $(6,10-12,15,19,21)$ instead of 1,4-benzodioxan-6-carbaldehyde.

N-Benzyl-3-[1-hydroxy-1-(5-phenyl-3,6-benzodioxanyl)methyl]-2-piperidone (24). Yield 31\%. ' $\mathrm{H}-\mathrm{NMR} \delta: 1.63-1.90(4 \mathrm{H}, \mathrm{m}, \beta-\mathrm{H}, \gamma-\mathrm{H}), 2.36-2.38(0.5 \mathrm{H}, \mathrm{m}, \alpha-\mathrm{H})$, 
$2.82-2.84(0.5 \mathrm{H}, \mathrm{m}, \alpha-\mathrm{H}), 3.10-3.24(2 \mathrm{H}, \mathrm{m}, \delta-\mathrm{H}), 4.30-4.77\left(5 \mathrm{H}, \mathrm{m},-\mathrm{C}_{\mathrm{H}}-\mathrm{Ph}\right.$, $\left.-\mathrm{O}-\mathrm{CH}_{2}-\mathrm{CH}-\mathrm{O}-\right), 5.00-5.20(2 \mathrm{H}, \mathrm{m},-\mathrm{C} \underline{\mathrm{H}}(\mathrm{OH})-,-\mathrm{OH}), 6.73-6.99(2 \mathrm{H}, \mathrm{m}, \mathrm{Ar}-\mathrm{H})$, 7.05-7.43 (5H, m, Ar-H), 7.69-7.70 (1H, m, Ar-H).

$N$-Benzyl-3-l1-hydroxy-1-(5-methoxymethyl-3,6-benzodioxanyl)melhyl $-2-$ piperidore (25). Yield $37 \%$. 'H-NMR $\delta: 1.64-1.73(4 \mathrm{H}, \mathrm{m}, \beta-\mathrm{H}, \gamma-\mathrm{H}), 2.53-2.54$ $(0.5 \mathrm{H}, \mathrm{m}, \alpha-\mathrm{H}), 2.80-2.82(0.5 \mathrm{H}, \mathrm{m}, \alpha-\mathrm{H}), 3.11-3.12(1 \mathrm{H}, \mathrm{m}, \delta-\mathrm{H}), 3.21-3.23(1 \mathrm{H}, \mathrm{m}, \delta$ $-\mathrm{II}), 3.42\left(1.5 \mathrm{H}, \mathrm{s}, \quad \mathrm{OCH}_{3}\right), 3.43\left(1.5 \mathrm{H}, \mathrm{s},-\mathrm{OCH}_{3}\right), 3.61-3.67(2 \mathrm{H}, \mathrm{m},-\mathrm{CH}-\mathrm{O}-), 4.05-1.15$ $\left(1 \mathrm{H}, \mathrm{m},-\mathrm{O}-\mathrm{CH}_{2}-\mathrm{CH}-\mathrm{O}-\right), 4.27-4.33\left(2 \mathrm{H}, \mathrm{m},-\mathrm{CH}_{2}-\mathrm{O}\right), 4.37-4.78(1.5 \mathrm{H}, \mathrm{m},-\mathrm{CH}(\mathrm{OH})-$, $\left.-\mathrm{CH}_{3}-\mathrm{Ph}\right), 5.14\left(0.5 \mathrm{H}, \mathrm{s},-\mathrm{C} \underline{\mathrm{H}}(\mathrm{OH})^{-}\right), 6.46(1 \mathrm{H}, \mathrm{s},-\mathrm{OH}), 6.83-7.01(3 \mathrm{H}, \mathrm{m}, \mathrm{Ar}-\mathrm{H})$, 7.18-7.37 (5H, m, Ar-H).

N-Benzyl-3-11-(5-ethoxymelhyl-3,6-henzodioxanyl)-1-hydroxymethyll-2piperidone (26). Yield 36\%, ${ }^{1} \mathrm{H}-\mathrm{NMR} \delta: 1.20-1.27\left(7 \mathrm{H}, \mathrm{m}, \beta-\mathrm{H}, \gamma-\mathrm{H},-\mathrm{CH}_{2}-\mathrm{CH}_{3}\right)$, $2.37-2.38(\mathrm{IH}, \mathrm{m}, \boldsymbol{\alpha}-\mathrm{H}), 3.21-3.23(2 \mathrm{H}, \mathrm{m}, \delta-\mathrm{H}), 3.54-3.71\left(6 \mathrm{H}, \mathrm{m},-\mathrm{O}-\mathrm{CH}_{2}-\mathrm{CH}_{2}-\mathrm{O}-\right.$, $\left.-\mathrm{O}-\mathrm{CH}_{2}-\mathrm{CH}_{3}\right), 4.04-4.13\left(1 \mathrm{H}, \mathrm{m},-\mathrm{O}-\mathrm{CH}_{2}-\mathrm{C} \underline{\mathrm{H}}-\mathrm{O}-\right), 4.28-4.31(1 \mathrm{H}, \mathrm{m},-\mathrm{C} \underline{\mathrm{H}}(\mathrm{OH})-)$, $4.52-4.67\left(2 \mathrm{H}, \mathrm{m},-\mathrm{CH}_{2}-\mathrm{Ph}\right), 6.47(1 \mathrm{H}, \mathrm{s},-\mathrm{OH}), 6.82-6.93(3 \mathrm{H}, \mathrm{m}, \mathrm{Ar}-\mathrm{H}), 7.25-7.37(5 \mathrm{H}$, $\mathrm{m}, \mathrm{Ar}-\mathrm{H})$.

N-Benzyl-3-[1-(5-benzyloxymethyl-3,6-benzodioxanyl)-1-hydroxymethyl]-2piperidone (27). Yield 64\%. ${ }^{1} \mathrm{H}-\mathrm{NMR} \delta: 1.63-1.73(4 \mathrm{H}, \mathrm{m}, \beta-\mathrm{H}, \gamma-\mathrm{H}), 2.52-2.53(1 \mathrm{H}$, $\mathrm{m}, \alpha-\mathrm{H}), 3.10-3.23(2 \mathrm{H}, \mathrm{m}, \delta-\mathrm{H}), 3.66-3.77\left(2 \mathrm{H}, \mathrm{m},-\mathrm{O}-\mathrm{CH}_{2}-\right), 4.06-4.15(1 \mathrm{H}, \mathrm{m}$, $-\mathrm{O}-\left(\mathrm{CH}_{2}-\mathrm{CH}-\mathrm{O}-\right), 4.28-4.32\left(2 \mathrm{H}, \mathrm{m},-\mathrm{O}-\mathrm{CH}_{2}-\right), 4.45-4.77\left(4 \mathrm{H}, \mathrm{m},-\mathrm{CH}_{2}-\mathrm{Ph},-\mathrm{CH}_{2}-\mathrm{Ph}\right)$, $5.15\left(1 \mathrm{H}, \mathrm{s},-\mathrm{CH}(\mathrm{OH})^{-}\right), 6.46(1 \mathrm{H}, \mathrm{s},-\mathrm{OH}), 6.82-6.93(2 \mathrm{H}, \mathrm{m}, \mathrm{Ar}-\mathrm{H}), 7.18-7.35(11 \mathrm{H}, \mathrm{m}$, $\mathrm{Ar}-\mathrm{H}$ ).

N-Benzyl-3-[1-hydroxy-1-(2-methoxy-3, 6-benzodioxanyl)methyl]-2-piperidone (28). Yield 43\%, 'H-NMR $\delta: 1.38-1.46(2 \mathrm{H}, \mathrm{m}, \beta-\mathrm{H}), 1.61-1.67(1 \mathrm{H}, \mathrm{m}, \gamma-\mathrm{H})$, $1.75-1.80(1 \mathrm{H}, \mathrm{m}, \gamma-\mathrm{H}), 2.62-2.69(1 \mathrm{H}, \mathrm{m}, \alpha-\mathrm{H}), 3.21-3.25(2 \mathrm{H}, \mathrm{m}, \delta-\mathrm{H}), 3.87(3 \mathrm{H}, \mathrm{s}$, $\left.-\mathrm{OCH}_{3}\right), 4.23-4.35\left(4 \mathrm{H}, \mathrm{m},-\mathrm{O}-\mathrm{CH}_{2}-\mathrm{CH}_{2}-\mathrm{O}-\right), 4.52\left(1 \mathrm{H}, \mathrm{d}, \mathrm{J}=15 \mathrm{~Hz},-\mathrm{CH}_{2}-\mathrm{Ph}\right), 4.73(1 \mathrm{H}$, d, J=15 Hz, $\left.-\mathrm{CH}_{2}-\mathrm{Ph}\right), 5.12\left(1 \mathrm{H}, \mathrm{d}, \mathrm{J}=10 \mathrm{~Hz},-\mathrm{CH}_{\mathrm{z}}-\mathrm{Ph}\right), 6.30(1 \mathrm{H}, \mathrm{s},-\mathrm{OH}), 6.54(1 \mathrm{H}, \mathrm{d}$, $\mathrm{J}=10 \mathrm{~Hz}, \mathrm{Ar}-\mathrm{H}), 7.01(1 \mathrm{H}, \mathrm{d}, \mathrm{J}=9 \mathrm{~Hz}, \mathrm{Ar}-\mathrm{H}), 7.23-7.37(5 \mathrm{H}, \mathrm{m}, \mathrm{Ar}-\mathrm{H})$.

N-Benzyl-3-(1-hydroxy-1-(3-methoxy-4,7-benzodioxanyl)methyll-2-piperidone (29). Yield 44\%. 'H-NMR $\delta: 1.32-1.33(1 \mathrm{H}, \mathrm{m}, \beta-\mathrm{H}), 1.61-1.80(3 \mathrm{H}, \mathrm{m}, \beta-\mathrm{H}, \gamma-\mathrm{H})$, $2.20(1 \mathrm{H}, \mathrm{s},-\mathrm{OH}), 2.45-2.54(1 \mathrm{H}, \mathrm{m}, \alpha-\mathrm{H}), 3.18-3.45(2 \mathrm{H}, \mathrm{m}, \delta-\mathrm{H}), 3.89\left(3 \mathrm{H}, \mathrm{s},-\mathrm{OCH}_{3}\right)$, $4.24-4.31\left(4 \mathrm{H}, \mathrm{m},-\mathrm{O}-\mathrm{CH}_{2}-\mathrm{CH}_{2}-\mathrm{O}^{-}\right), 4.53-4.75\left(3 \mathrm{H}, \mathrm{m},-\mathrm{CH}(\mathrm{OH})^{-},-\mathrm{CH}_{2}-\mathrm{Ph}\right), 6.50-6.63$ $(2 \mathrm{H}, \mathrm{m}, \mathrm{Ar}-\mathrm{H}), 7.22-7.37(5 \mathrm{H}, \mathrm{m}, \mathrm{Ar}-\mathrm{H})$.

N-Benzyl-3-11-hydroxy-1-(2-methoxy-4,7-benzodioxanyl)methyl-2-piperidone (30). Yield 70\%, $\mathrm{H}-\mathrm{NMR} \delta: 1.37-1.65(4 \mathrm{H}, \mathrm{m}, \beta-\mathrm{H}, \gamma-\mathrm{H}), 2.52-2.57(0.5 \mathrm{H}, \mathrm{m}, \alpha-\mathrm{H})$, $2.89-2.92(0.5 \mathrm{H}, \mathrm{m}, \alpha-\mathrm{H}), 3.12-3.17(1 \mathrm{H}, \mathrm{m}, \delta-\mathrm{H}), 3.20-3.26(1 \mathrm{H}, \mathrm{m}, \delta-\mathrm{H}), 3.73(3 \mathrm{H}, \mathrm{s}$, $\left.-\mathrm{OCH}_{3}\right), 3.91(0.5 \mathrm{H}, \mathrm{d}, \mathrm{J}=6,-\mathrm{OH}), 4.20-4.49\left(4 \mathrm{H}, \mathrm{m},-\mathrm{O}-\mathrm{CH}_{2}-\mathrm{CH}_{2}-\mathrm{O}-\right), 4.49-4.57(1 \mathrm{H}, \mathrm{m}$, $\left.-\mathrm{CH}_{2}-\mathrm{Ph}\right), 4.69-4.79\left(1 \mathrm{H}, \mathrm{ml},-\mathrm{CH}_{2}-\mathrm{Ph}\right), 5.19(0.5 \mathrm{H}, \mathrm{d}, \mathrm{J}=9 \mathrm{~Hz},-\mathrm{CH}(\mathrm{OH})-), 5.69(0.5 \mathrm{H}$, $\mathrm{dd}, \mathrm{J}=4,6 \mathrm{~Hz},-\mathrm{CH}(\mathrm{OH})-), 6.25(0.5 \mathrm{II}, \mathrm{s}, \mathrm{Ar}-\mathrm{H}), 6.39(1 \mathrm{H}, \mathrm{d}, \mathrm{J}=3 \mathrm{~Hz}, \mathrm{Ar}-\mathrm{H}), 7.04(1 \mathrm{H}, \mathrm{d}$, $\mathrm{J}=10 \mathrm{~Hz}, \operatorname{Ar}-\mathrm{H}), 7.23-7.34(5 \mathrm{H}, \mathrm{m}, \mathrm{Ar}-\mathrm{H})$.

\section{Lettuce seedling test}

The bioassay using lettuce seedlings (Jastuca sativa L.c.v. Sacramento) was performed according to the method described previously (Tsukada et al., 1999). After 7 days of incubation, the length of the primary roots was measured and the emergence of 
the visible lateral roots was inspected. The growth rates were calculated as percentages of the averaged lengths of primary roots of treated plants to those of controls (deionized water). A primary root was considered responsive when it contained at least one lateral root. In controls the percentage of emerged lateral roots was less than $2 \%$.

\section{RESULTS AND DISCUSSIONS}

\section{Synthesis}

Substituted 1,4-benzodioxancarbaldehydes, key intermediates for synthesis of $N$-benzyl-3-[1-hydroxy-1-(substituted 3,6-benzodioxanyl)methyl]-2-piperidones, were prepared according to the route depicted in Fig. 2 and 3. 2-Substituted-1,4-benzodjoxan-6-carbaldehydes 6, 10-12 (Fig. 2) and 7-methoxy-1,4-benzodioxan-6-carbaldehyde (15, Fig. 3) were synthesized by the procedure previously used for the synthesis of haedoxan A analogs (Taniguchi et al., 1992). Ketone $\mathbf{3}$ was prepared from 4-benzyloxy-3 hydroxybenzaldehyde (2, Kessar et al., 1983) and phenacyl bromide. Conversion

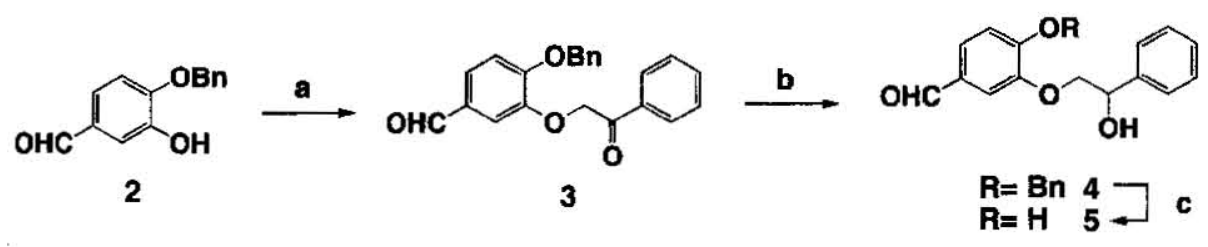<smiles>O=Cc1ccc2c(c1)OCC(c1ccccc1)O2</smiles>

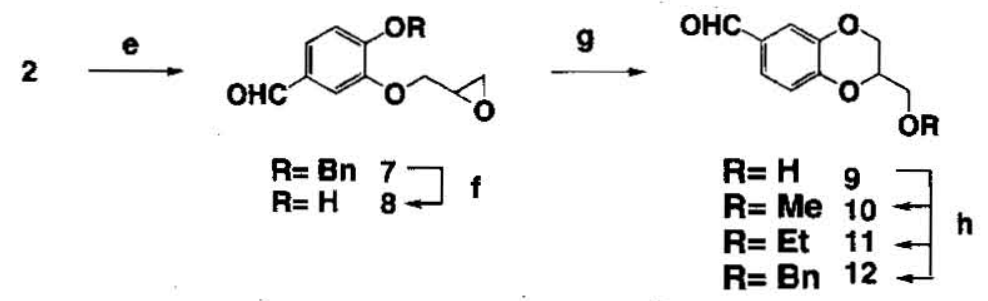

Fig. 2. Synthesis of 2-substituted-1,4-benzodioxan-6-carbaldehydes

Reagents and conditions: (a) phenacy] bromide, $\mathrm{K}_{2} \mathrm{CO}_{3}, 18$-crown-6, $\mathrm{CH}_{i 1} \mathrm{CN}$, room temperature; (b) 1) trimethyl orthoformate, $\mathrm{NH}_{4} \mathrm{Cl}$, THF : $\mathrm{MeOH}=3: 1$, reflux, 2) $\mathrm{NaBH}_{4}, \mathrm{THF}$ : $\mathrm{McOH}=4: 1,0^{\circ} \mathrm{C}$ to room temperature, 3) $2 \mathrm{~N} \mathrm{HCl}$ solution, room temperature; (c) $\mathrm{Pd} / \mathrm{C}$. EtoAc : $\mathrm{McOH}=1: 1$, room temperature; (d) pyridinium $p$-tolucnesulfonatc, toluene, reflux; (e) 1-bromo-2,3-epoxypropant, $\mathrm{NaH}, \mathrm{DMF}, 0^{\circ} \mathrm{C}$ to room tempcrature; (f) $\mathrm{Pd} / \mathrm{C}$, Et.OAc, room temperature; (g) $\mathrm{K}_{2} \mathrm{CO}_{1}, 18$ crown-6, $\mathrm{CH}_{3} \mathrm{CN}$, room temperature; (h) alkyl bromide or iodide, $\mathrm{NaOH}$, tetrabutylammonium hyctrogen sulfate, water- $\mathrm{CH}_{2} \mathrm{Cl}_{2}=1: 1$, room temperature. 

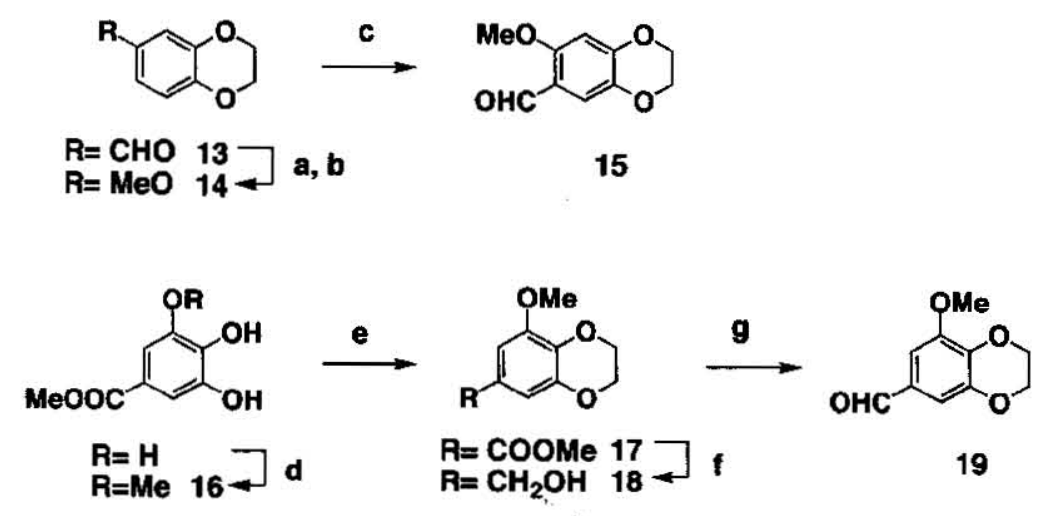

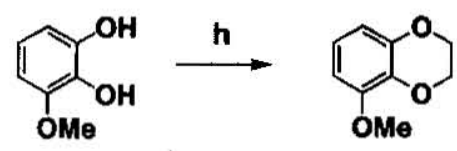

20

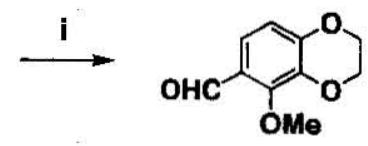

21

Fig. 3. Synthesis of 1,4-benzodioxan-6-carbaldehydes with a methoxy group Reagents and conditions: (a) 1) $m$-chloroperbenzoic acid, $\mathrm{KF}, \mathrm{CH}_{2} \mathrm{Cl}_{2}$, $0^{\circ} \mathrm{C}$-room temperature, 2) aq. $\mathrm{NaOH}$, room temperature; (b) $\mathrm{K}_{2} \mathrm{CO}_{n}, \mathrm{MeI}$, acetone, reflux; (c) $\mathrm{POCl}_{3}, \mathrm{DMF}, 100^{\circ} \mathrm{C}$; (d) $\mathrm{NaOH}$, sodium tetraborate solution, $\mathrm{Me}_{2} \mathrm{SO}_{4}$ room temperature; (e) $\mathrm{K}_{2} \mathrm{CO}_{3}, 1,2$-dibromoethane, acetone, reflux; (f) $\mathrm{LiAlH}_{4}, \mathrm{THF}, 0^{\circ} \mathrm{C}$ to room temperature; (g) DMSO, $(\mathrm{COCl})_{2}, \mathrm{Et}_{3} \mathrm{~N}, \mathrm{CH}_{2} \mathrm{Cl}_{3},-78^{\circ} \mathrm{C}$; (h) $\mathrm{K}_{2} \mathrm{CO}_{3}, 1,2$-dibromoethane, acetone, reflux; (i) $\mathrm{POCl}_{3}, \mathrm{DMF}, 100^{\circ} \mathrm{C}$

of $\mathbf{3}$ into alcohol $\mathbf{4}$ was executed by the following three-step sequence: (i) acetalization of the formyl group; (ii) reduction of the keto group by sodium borohydride; (iii) deacetalization under acidic: conditions. Successive debenzylation of $\mathbf{4}$ with palladium on carbon afforded phenol $\mathbf{5}$, which was cyclized to 2-phenyl-1,4-benzodioxan 6 by treatment with a catalytic amount of $p$-toluenesulfonic acid.

Reaction of $\mathbf{2}$ with 1-bromo-2,3-epoxypropane gave epoxide $\mathbf{7}$. Debenzylation of $\mathbf{7}$ with palladium on carbon followed by cyclization with potassium carbonate provided 2-hydroxymethyl-1,4-benzodioxan 9. Alkylation of $\mathbf{9}$ with the corresponding alkyl bromide or iodide using tetrabutylammonium hydroxide as a base afforded alkyl ethers 10-12.

1,4-Benzodioxancarbaldehydes having a methoxy group at the different position on the benzene ring were prepared by three procedures as shown in Fig. 3. The Bayer-Villiger oxidation of 1,4-benzodioxan-6-carbaldehyde (13) with $m$-chloroperbenzoic acid followed by alkaline hydrolysis afforded 6-hydroxy-1,4-benzodioxan, which was alkylated with methyl iodide and potassium carbonate to yield 6-methoxy-1,4-benzodiox- 


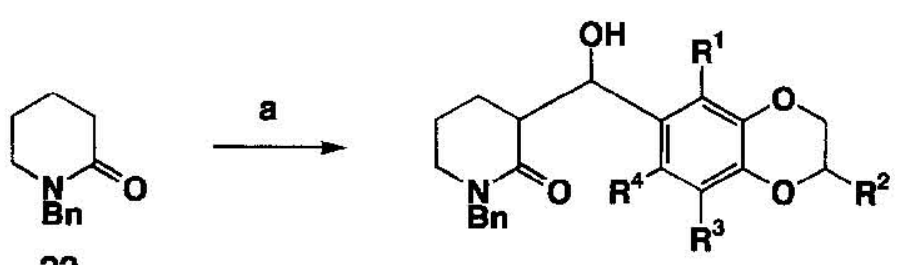

22

\begin{tabular}{|c|c|c|c|c|}
\hline$R^{1}$ & $\mathbf{R}^{2}$ & $\mathbf{R}^{3}$ & $\mathrm{R}^{4}$ & No. \\
\hline H & H & H & H & 23 \\
\hline H & $\mathbf{P h}$ & H & H & 24 \\
\hline $\begin{array}{l}\mathrm{H} \\
\mathrm{H}\end{array}$ & $\mathrm{CH}_{2} \mathrm{OMe}$ & $\begin{array}{l}\mathrm{H} \\
\mathrm{H}\end{array}$ & $\begin{array}{l}H \\
H\end{array}$ & $\begin{array}{l}25 \\
26\end{array}$ \\
\hline H & $\mathrm{CH}_{2} \mathrm{OBn}$ & H & H & 27 \\
\hline $\begin{array}{c}\text { MeO } \\
\mathbf{H} \\
\mathbf{H}\end{array}$ & $\begin{array}{l}\mathbf{H} \\
\mathbf{H} \\
\mathbf{H}\end{array}$ & $\underset{\text { MeO }}{H}$ & $\underset{M e O}{H}$ & $\begin{array}{l}28 \\
29 \\
30\end{array}$ \\
\hline
\end{tabular}

Fig. 4. Synthesis of $N$-benzyl-3-[1-(3,6-benzodioxanyl)-1-hydroxymethyl]-2-piperidone and related compounds

Reagents and conditions: (a) lithium diisopropylamide, aldehyde, THF, $-78^{\circ} \mathrm{C}$.

an (14). Regioselective formylation by the Vilsmeier reaction gave aldehyde $\mathbf{1 5}$ in a $78 \%$ yield. Treatment of methyl 3,4,5-trihydroxybenzoate with dimethyl sulfate in the presence of sodium tertaborate and sodium hydroxide (Scheline, 1966) afforded 3-methoxy-5-methoxycarbonylcatechol (16), which was cyclized to 1,4-benzodioxan 17 with 1,2-dibromoethane and potassium carbonate. Reduction of $\mathbf{1 7}$ with lithium aluminum hydride, followed by Swern oxidation, gave aidehyde 19. 5-Methoxy-1,4-benzodioxan-6-carbaldehyde (21) was prepared from 3-methoxycatechol. Cyclization of 3-methoxycatechol to 5-methoxy-1,4-benzodioxan (20) was accomplished in the same manner as that used for compound 17. Formylation of $\mathbf{2 0}$ using the same Vilsmeier reaction employed in the synthesis of $\mathbf{1 5}$ afforded the desired aldehyde $\mathbf{2 1}$.

$N$-Benzyl-2-piperidone (22) was prepared according to the procedure reportcd previously (Tsukada et al., 1999). The lithium enolate of $\mathbf{2 2}$ generated with lithium disopropylamide (LDA) in THF was treated with various 1,4-benzodioxan-6-carbaldehydes to give a mixture of erythro and threo aldol products 23-30 (Fig. 4). An attempt was made to separate two diastereomeric isomers of $\mathbf{2 3}$. In this aldol reaction, the thermodynamically preferred erythro-23 was obtained as the major product when the reaction mixture was stirred at $-78^{\circ} \mathrm{C}$ for 3 hours, while under a shorter reaction time (1 hour) threo-23 was formed in preference to the erythro isomer. Thus, the erythroand threo-23 were isolated from the reaction mixture under the former and the latter 
conditions, respectively, using column chromatography on silica gel. Confirmation of the stereochemistry of the erythro- and threo-23 was based on the observation of their 'H-NMR spectra which showed the different coupling constant between $3-\mathrm{H}$ and 1 '- $\mathrm{H}$ ( $\delta$ $5.15 \mathrm{ppm}, \mathrm{J}_{3-1}=3 \mathrm{~Hz}$ for $\mathrm{I}^{\prime}-\mathrm{H}$ of eryethro; and $\delta 4.64 \mathrm{ppm}, \mathrm{J}_{3: \mathrm{l}}=10 \mathrm{~Hz}$ for $1^{\prime}-\mathrm{H}$ of threo) (House et al., 1973).

\section{Lateral root-inducing activity}

Table 1 shows the effect of a series of $N$-benzyl-2-piperidones with a 1,4-benzodioxan moiety at the 3 position on the growth of lettuce seedlings. Most of the compounds inhibited the growth of primary root less than $50 \%$ of the control value at $100 \mathrm{ppm}$. In seedlings treated with $100 \mathrm{ppm}$ of the 1,4-benzodioxan analog $\mathbf{2 3}$, which is a 1:1 mixture of erythro and threo, all of the primary roots formed lateral roots, however, its activity rapidly decreased at $10 \mathrm{ppm}$. Since there was no significant difference in lateral root-inducing activity between the erythro- and threo-23, the activity of other benzodioxan analogs was evaluated for the diastereomeric mixtures.

A modification was first made by introducing a pheryl (24), methoxymethyl (25), ethoxymethyl (26) or benzyloxymethyl (27) group at the 2-position on the 1,4-bezodioxan ring of compound 23. Compounds 24-26 had almost the same activity as that of non-substituted benzodioxan analog $\mathbf{2 3}$, while the benzyloxymethyl analog $\mathbf{2 7}$ possessed slightly higher activity than $\mathbf{2 3}$, showing $16 \%$ emergence of lateral roots even at $1 \mathrm{ppm}$. These results indicate that a substituent at the 2-position on the 1,4-bezodioxan ring did not significantly involve in lateral root-inducing activity.

The introduction of a methoxy group at the 5-(28) or 8-(29) position on the 1,4-benzodioxan ring drastically decreased the activity compared with that of $\mathbf{2 3}$, whereas the stirnulation of the primary root growth was observed by treatment of these compounds at low concentrations. The 2 -methoxy-4,7-benzodioxan analog $\mathbf{3 0}$ showed lateral root-inducing activity comparable to that of $\mathbf{2 3}$. It is noteworthy that the activity was greatly affected by a methoxy group adjacent to the 1,4-dioxane ring.

Table 1. Effects of $N$-benzyl-3-[1-hydroxy-1-(substituted-3,6-benzodioxanyl)methyll-2-piperidones on root growth of lettuce seedlings

\begin{tabular}{|c|c|c|c|c|c|c|c|}
\hline \multirow[b]{2}{*}{ No. } & \multirow[b]{2}{*}{ Conc. (ppm) } & \multicolumn{3}{|c|}{$\begin{array}{l}\text { Growth rate of primary rout } \\
\text { (\% of control) }\end{array}$} & \multicolumn{3}{|c|}{$\begin{array}{l}\text { Emergence of lateral root } \\
(\%)\end{array}$} \\
\hline & & 100 & 10 & 1 & 100 & 10 & 1 \\
\hline $23^{i}$ & & 12 & 54 & 106 & 100 & 3 & 0 \\
\hline erythro-23 & & 14 & 44 & 98 & 100 & 30 & 5 \\
\hline threo-23 & & 24 & 72 & 103 & 100 & 6 & 0 \\
\hline 24 & & 29 & 50 & 86 & 100 & 21 & 0 \\
\hline 25 & & 30 & 77 & 70 & 100 & 2 & 0 \\
\hline 26 & & 50 & 93 & 100 & 100 & 15 & 0 \\
\hline 27 & & 28 & 77 & 78 & 100 & 16 & 16 \\
\hline 28 & & 40 & 131 & 127 & 37 & 0 & 0 \\
\hline 29 & & 50 & 121 & 101 & 0 & 0 & 0 \\
\hline 30 & & 13 & 54 & 96 & 100 & 11 & 0 \\
\hline
\end{tabular}

- A 1:1 mixture of erythro and threo 
Although compounds $\mathbf{2 3}$ and $\mathbf{2 7}$ were less active than compound $\mathbf{1}$, which is the most effective among the compounds tested so far, this new series of 2-piperidones with a 1,4-benzodioxan ring is worthy of further investigation toward the development of a structurally novel class of plant growth regulators. More detailed studies on the structure-activity relationships are under way.

\section{REFERENCES}

Hansel, R., J. Schulz and A. Pelter 1980 Structure of silybin: synthetic studies. J. Chem. Soc., Chem. Commun., 195-196

House, H. O., D. S. Crumrine, A. Y. Teranishi and H. D. Olmstead 1973 Chernistry of carbartions. XXII Use of metal complexes to control the aldol condensation. J. Am. Chem. Soc, 95: 3310-3324

Kessar, S. V., Y. P. Gupta, T. Mohammad, M. Goyal and K. K. Sawal 1983 Regioselective mono-O-alkylation of some pyrocatechoxide dianions. J. Chem. Soc, Chem. Commun., 400-401

Ray, A. B., S. K. Chattopadhyay, C. Konno and H. Hikino 1980 Structure of cleomiscosin A, a coumarino-lignoid of Cleome viscosa sceds. Tetrahedron Lett. $4477-4480$

Scheline, R. R. 1966 A rapid synthesis of 3-O-methylgallic acid. Acta Chem. Scand., 20: 1182-1183

Taniguchi, E., K. Imamura, F. Ishibashi, T. Matsui, and A. Nishio 1989 Structure of the novel insecticidal sesquilignan, haedoxan A. Agric. Biol. Chem., 53: 631-643

Taniguchi, E., S. Yartauchi, S. Nagata and T. Ohnishi 1992 Syntheses of 2-substituted 6/7-methoxy-1,4-benzodioxan-7/6-carbaldehydes. Biosci. Biotech Biochem., 56: 630-635

Tsukada, H., T. Itamura, R. Ishii, E. Taniguchi and E. Kuwano 1999 Synthesis and lateral root-inducing activity of $N$-benzyl-3-substituted-2-piperidones. J. Fac. Agr, Kyushu Lnio., 44: 119-126 (1999)

Woo, W. S., S. S. Kang, H. Wagner and M. Chari 1978 Die Struktur von Americanin, eincm neuen Neolignan aus Phytolacca americana. Tetrahedron Lett., 32393242 